DE-FC26-04NT42277

\title{
Surface Plasmon Enhanced Phosphorescent Organic Light Emitting Diodes
}

\author{
Guillermo C. Bazan and Alexander A. Mikhailovsky \\ Institute for Polymers and Organic Solids, University of California Santa Barbara \\ Santa Barbara, CA 93106-9510 \\ Final Report
}

\section{Project Objectives and Technical Approach Summary:}

The objective of the proposed work was to develop the fundamental understanding and practical techniques for enhancement of Phosphorescent Organic Light Emitting Diodes (PhOLEDs) performance by utilizing radiative decay control technology. Briefly, the main technical goal is the acceleration of radiative recombination rate in organometallic triplet emitters by using the interaction with surface plasmon resonances in noble metal nanostructures. Increased photonic output will enable one to eliminate constraints imposed on PhOLED efficiency by triplet-triplet annihilation, triplet-polaron annihilation, and saturation of chromophores with long radiative decay times. Surface plasmon enhanced (SPE) PhOLEDs will operate more efficiently at high injection current densities and will be less prone to degradation mechanisms. Additionally, introduction of metal nanostructures into PhOLEDs may improve their performance due to the improvement of the charge transport through organic layers via multiple possible mechanisms ("electrical bridging" effects, doping-like phenomena, etc.).

SPE PhOLED technology is particularly beneficial for solution-fabricated electrophosphorescent devices. Small transition moment of triplet emitters allows achieving a significant enhancement of the emission rate while keeping undesirable quenching processes introduced by the metal nanostructures at a reasonably low level. Plasmonic structures can be introduced easily into solution-fabricated PhOLEDs by blending and spin coating techniques and can be used for enhancement of performance in existing device architectures. This constitutes a significant benefit for a large scale fabrication of PhOLEDs, e.g. by roll-to-roll fabrication techniques.

Besides multieexciton annihilation, the power efficacy of PhOLEDs is often limited by high operational bias voltages required for overcoming built-in potential barriers to injection and transport of electrical charges through a device. This problem is especially pronounced in solution processed OLEDs lacking the accuracy and precision of fabrication found in their small molecule counterparts. From this point of view, it seems beneficial to develop materials allowing reduction of the operation bias voltage via improvement of the charge injection. The materials sought have to be compatible with solution-based fabrication process and allow easy incorporation of metal nanostructures. 


\section{DISCLAIMER}

This report was prepared as an account of work sponsored by an agency of the United States Government. Neither the United States Government nor any agency thereof, nor any of their employees, makes any warranty, express or implied, or assumes any legal liability or responsibility for the accuracy, completeness, or usefulness of any information, apparatus, product, or process disclosed, or represents that its use would not infringe privately owned rights. Reference herein to any specific commercial product, process, or service by trade name, trademark, manufacturer, or otherwise does not necessarily constitute or imply its endorsement, recommendation, or favoring by the United States Government or any agency thereof. The views and opinions of authors expressed herein do not necessarily state or reflect those of the United States Government or any agency thereof. 


\section{Background information}

Radiative decay engineering technology relies on the modification of the density of photonic states in systems imposing boundary conditions on the emitted electromagnetic field. According to Einstein's formula, the rate of spontaneous emission is proportional to the density of states for electromagnetic field (photonic states density). Metal nanoparticles (NPs) exhibit a significant modification of the photonic states density in the near field zone around their surface. An emissive molecule situated in the vicinity of NPs surface will emit light faster than in free space. Additional enhancement of the spontaneous emission rate can be observed if the emission is resonant with the surface plasmon (SP) oscillations in the metal nanostructure. However, besides acceleration of the radiative transitions, metal surface can increase the rate of energy losses due to the energy transfer between the excited chromophore and the metal. The primary emission quenching mechanism is dipole-dipole in nature and decays faster when the molecule is moved away from the metal surface than the enhancement of the emissive processes. Triplet emitters are expected to have smaller quenching radius due to their small oscillator strength. Thus, they can be situated in the vicinity of the metal surface where the emission rate enhancement is significant, while keeping the increase of the energy losses at acceptable levels. Therefore, they may have a significant advantage over regular, singlet fluorophores in radiative decay technology applications.

Acceleration of the light emission by triplet chromophores in PhOLEDs may allow one to overcome the negative influence of the multiexciton annihilation processes (triplet-triplet annihilation (TTA), in particular) by creation of competing channels for energy dissipation via emission of light quanta. In its turn, reduction of the TTA-related energy losses enables efficient operation of OLEDs in the high current density regime typical for lightning applications. It is worth noting that in many cases the increase of the light emission rate will not improve the peak quantum efficiency of the device because the chromophores used in the light emitting layer (LEL) are already nearly $100 \%$ efficient.

The general recipe for the implementation of the radiative decay control technology requires placement of the emissive molecules in the vicinity of metal NPs while the emission band is being resonant with SP band in NPs. It is desirable to have an "insulating layer" of transparent dielectric or molecules on the metal surface to prevent direct contact between the metal and the emissive molecules and the resulting quenching of the luminescence. In solution processed OLEDs, metal NPs can be embedded directly into LEL, e.g., by admixing them into the LEL casting solution. Or they can be incorporated into the surface of layers adjacent to LEL, such as electron and hole transport layers (ETL and HTL, respectively). In the latter case, the region of the emission rate enhancement is expected to be small and limited to the area adjacent to the interface between LEL and charge transport layer. Obviously, technological issues such as compatible solubility and miscibility of NPs and organic materials have to be addressed as well.

\section{Task 3. Research and development of SPE PhOLEDs*}

We have focused on the achievement of Milestone I primarily during the Budget Period 1 (BP1). As a model system, we used green-emitting PhOLEDs with the general architecture ITO/PEDOT:PSS+AuNPs/Ir(mppy $)_{3}: \mathrm{PVK}: \mathrm{PBD} / \mathrm{Ba} / \mathrm{Al}$ shown in the Figure 1 . The green phosphorescent complex $\operatorname{Ir}(\mathrm{mppy})_{3}$ has a good overlap of the emission band with the surface

\footnotetext{
* The discussion of the project tasks is laid out not in the numerical order, but in the order of their importance.
} 
plasmon (SP) resonance in small ( $<50 \mathrm{~nm}$ ) gold spherical nanoparticles (NPs) which ensured an efficient photonic interaction between the emissive molecules and SPs. Commercially available, water-soluble Au NPs were incorporated into the hole transport layer (Baytron PEDOT:PSS) by mixing the stock solutions prior to the HTL deposition. Addition of metal NPs to the HTL has a pronounced effect on the device performance in the high injection current

$\mathrm{Ba} / \mathrm{Al}$ cathode

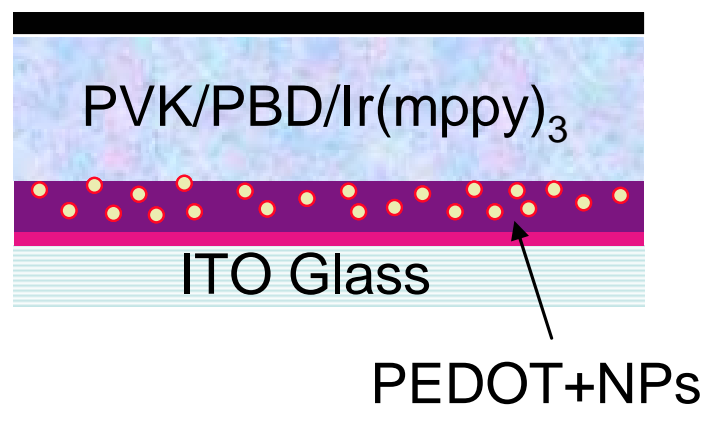

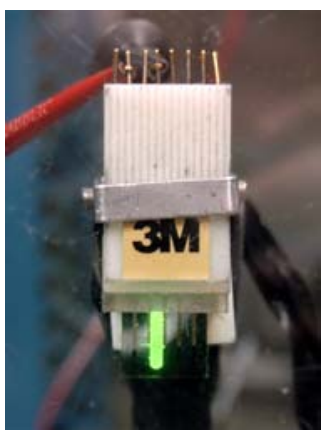

Figure 1. Layout and a photograph of green SPE PhOLED density regime (Figure 2). Despite the maximum efficiency of the devices was not affected significantly by the presence of NPs, a large increase of the operational current density has been reported. It can be quantified by using $\mathrm{J}_{0.5}$ parameter - the current density corresponding to $50 \%$ rolloff of the device efficiency. Typically, SPE PhOLEDs exhibit 4-5-fold increase of $\mathrm{J}_{0.5}$, corresponding to approximately two-fold increase of the triplet exciton recombination rate. High operational current density translates into the high value of luminance. In SPE PhOLEDs based on Ir(mppy) $)_{3}$, we obtained brightness close to $80000 \mathrm{Cd} / \mathrm{m}^{2}$. Insignificant variation of the maximum device efficiency in the small current density regime was attributed to the high internal efficiency of the triplet emitter which cannot be improved further by faster radiative processes. Experiments with Au NPs embedded into the water-soluble electron transport layer (ETL) demonstrated similar behavior (not shown here). The results have been reproduced in a very large number of devices and are statistically valid. Thus, we have successfully achieved Milestone I "Successful demonstration of SPE PhOLED technology”.

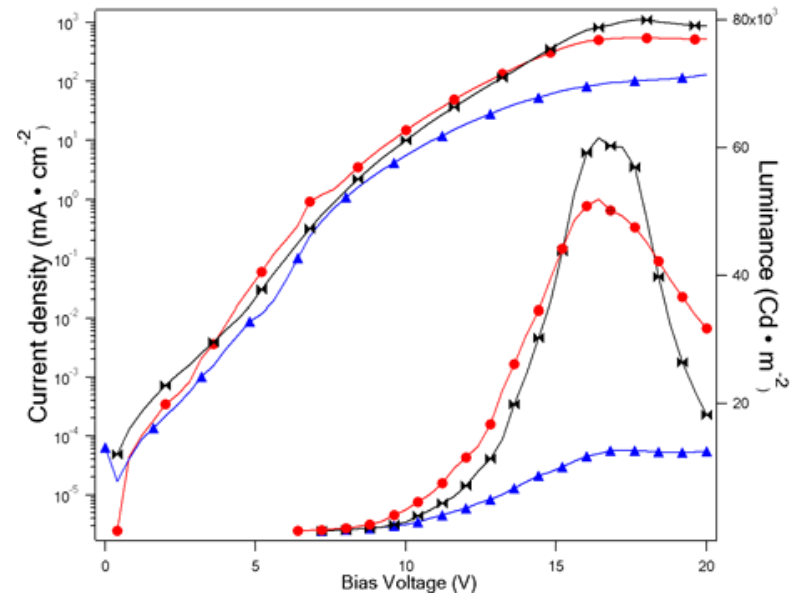

(a)

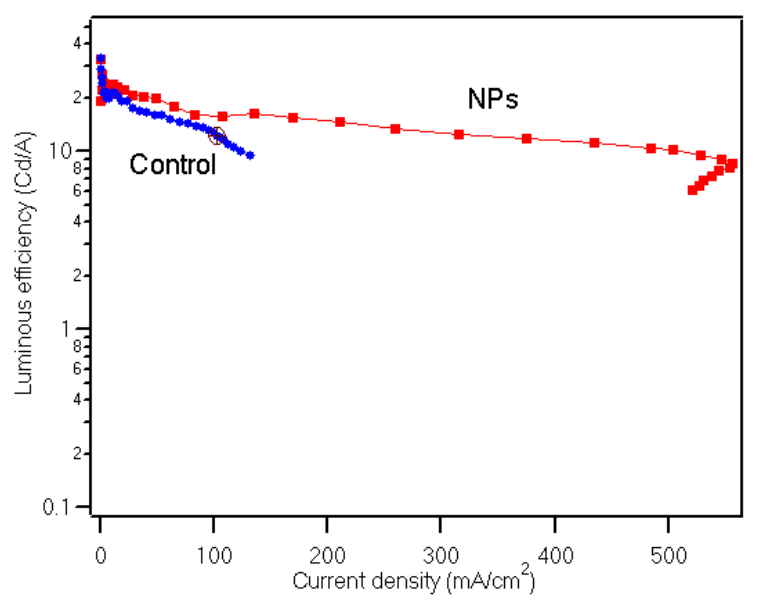

(b)

Figure 2. (a) J-V and L-V for Au NP-doped Ir(mppy) ${ }_{3}$ - based PhOLEDs. (b) PhOLEDs efficiency as a function of injection current density. 


\section{Blue-emitting SPE PhOLEDs}

As a first step in the development of all-triplet white-emitting PhOLEDs, we attempted to fabricate NP-doped blue emitting PhOLEDs and study the influence of metal NPs on their performance. These efforts were stimulated by the fact that blue-emitting electrophosphorescent

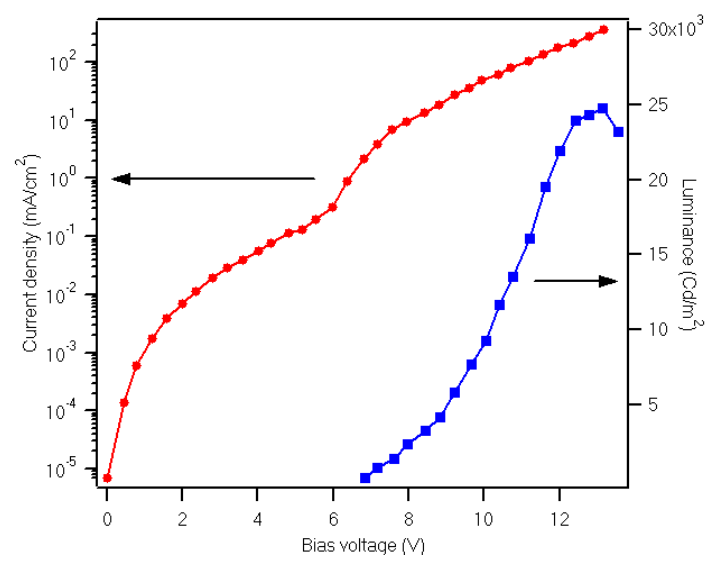

Figure3. J-V and L-V curves for $20 \mathrm{~nm} \mathrm{Au}$ NP-doped FirPIC SPE PhOLEDs.

devices have the shortest life-time and generally are less efficient than their green- and redemitting counterparts. The basic structure for the blue-emitting devices was ITO/PEDOT:PSS(+Au NPs)/FIrpic:PVK:OXD7/Ba/Al. Ir(III)bis [(4,6-di-fluoropheny)pyridinato- $\mathrm{N}, \mathrm{C}^{2}$ ] $]$ picolinate (FIrPic) is a wellknown and efficient blue triplet emitter. It was obtained from Prof. Mark Thomson group at the University of South California, along with the electron transport material 1,3-bis[2-(4-tertbutylphenyl)-1,3,4-oxadiazol-5-yl]benzene (OXD-7). Poly(9-vinylcarbazole) (PVK) was used as a host material. We have tested various host and electron transport materials, such as 4,4'-bis(9-carbazolyl)-2,2'-biphenyl (CBP) and 2-(biphenyl-4-yl)-5-(4-tert-butylphenyl)-1,3,4oxadiazole (t-Bu-PBD), respectively. However, the use of these materials led to poorer device performance, if compared to the PVK/OXD-7 matrix. FIrpic-based PhOLEDs turned out to be very sensitive to the annealing of HTL and polymer layers. In properly annealed samples, we observed luminous efficiency close to $14 \mathrm{Cd} / \mathrm{A}$ and peak luminance of about $14000 \mathrm{Cd} / \mathrm{m}^{2}$. Doping of the HTL with metal NPs led to a significant increase of the device luminance. Interestingly, both Ag and Au NPs lead to the increase of the PhOLEDs efficiency in the high current density regime. The luminance value achieved in NP-doped, blue-emitting devices exceeds $25000 \mathrm{Cd} / \mathrm{m}^{2}$ (Figure 3) which is the record-high value for the solution-fabricated FIrPIc-Based devices.

\section{White-light emitting Devices.}

We investigated two strategies for the fabrication of white emitting PhOLEDs which can be used as a platform for the SPE PhOLED technology. White emission can be achieved in a single layer solution-fabricated OLED using either a combination of singlet and triplet emitters or triplet emitters only for generation of RGB spectral components. In the first configuration, host material can serve as a short-wavelength light emitter, provided that the energy transfer to guest chromophores is not complete.

Hybrid, singlet-triplet white OLEDs fabricated in our group utilized PFO as a host material which also served as source of the blue light. Green spectral component was generated by the poly(9,9 '-dioctylfluorene-co-benzothiadiazole) (F8BT), which is an efficient singlet chromophore. The red component was generated by using an, in-house synthesized Ir-based triplet emitter. These devices exhibited moderate luminous efficiency of $\sim 7-9 \mathrm{Cd} / \mathrm{A}$, with perfect color quality (CIE color coordinates $(0.33,0.33)$ ) in the low current density regime. However, there is a significant color shift in the high current density regime, due to the decrease of the 
triplet emitter efficiency caused by multiexciton non-radiative recombination processes. Also, despite the fact that hybrid devices demonstrated an increase of the operational current densities upon addition of metal NPs, the efficiency of NP-doped OLEDs was lower than that of the control devices. The decrease of the efficiency is explained by quenching of singlet emitters in the presence of metal particles. This problem can be avoided in all-triplet white PhOLEDs, since the efficiency of the chromophore quenching is proportional to its oscillator strength which is low in phosphorescent materials.

To create a test platform for the implementation of SPE PhOLED technology in whiteemitting devices, we developed a device architecture where all spectral components are

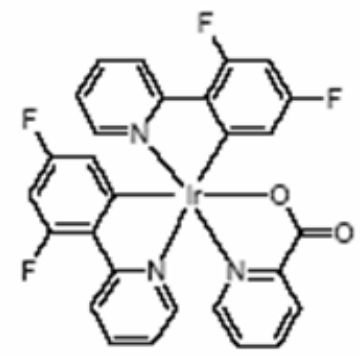

FIrPic

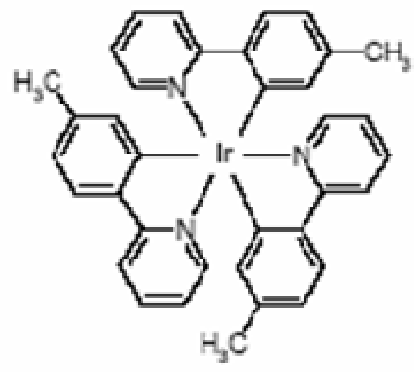

Ir (mppy) 3

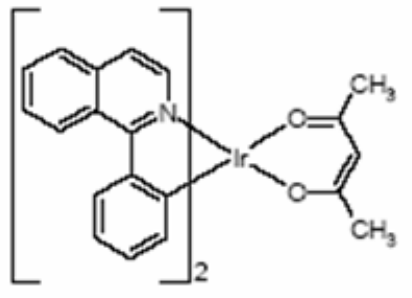

$\operatorname{Ir}(\mathrm{piq})$

Figure 4 Triplet emitters used in fabrication of white PhOLEDs.

generated by phosphorescent guest molecules. The chromophores used in these devices are FIrPiC (Blue), Ir(mppy) $)_{3}$ (Green), and Ir(piq) (Red) (See Figure 4 for the chemical structures). PVK was used as a host material, and OXD-7 was used to ensure good electron transport in the polymer layer. The components were mixed together and deposited by spin coating as a single layer on top of a PEDOT:PSS-coated ITO glass. A Ba/Al cathode was evaporated on top of the resulting structure. Water soluble metal NPs can be added to the HTL similar to the colored PhOLED structure shown in the Figure 1. All chromophores used in the all-triplet device architecture are nearly $100 \%$ efficient internally in single color devices, thus we anticipated achieving high internal quantum efficiency in white emitting devices as well. The typical results of the all-triplet device testing are shown in the Figure 5. The maximum external quantum

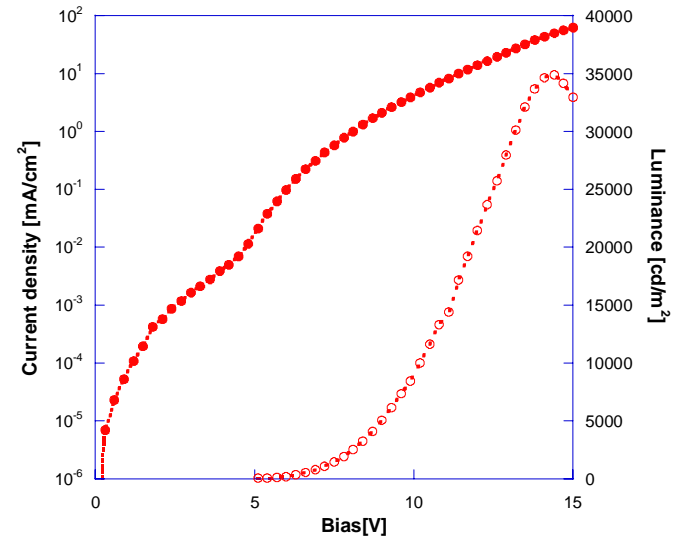

(a)

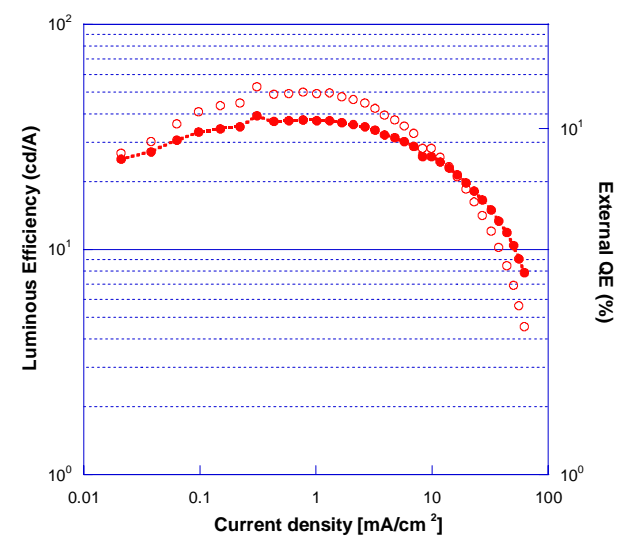

(b)

Figure 5. (a) JLV curves of the all-triplet white PHOLEDs; (b) Luminous and external quantum efficiencies as a function of the current density. 
efficiency is approximately $14 \%$, corresponding to $~ 70-80 \%$ internal quantum efficiency. The power efficacy of the devices varies between $11-17 \mathrm{~lm} / \mathrm{W}$ depending on the color coordinates of the emission which can varies by adjusting the ratio of RGB LEL components. The best CIE color coordinates achieved in these PhOLEDs are $(0.33,0.35)$ with corresponding power efficacy of 11-12 Lm/W. A series of devices of devices with power efficacy of 15-17 Lm/W had CIE color coordinates $(0.34,0.45)$.

Metal NPs had an effect on the device performance similar to that observed in monochromatic devices (See Figure 6). However, it's was noticed that addition of NPs to the devices leads to a significant improvement of the performance only if the parameters of control devices are far from optimal due to the poor interfacial properties limiting the injection of charge

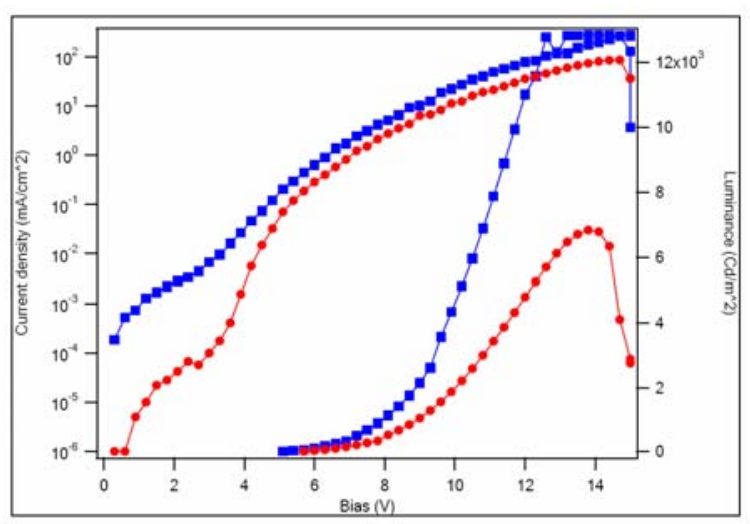

(a)

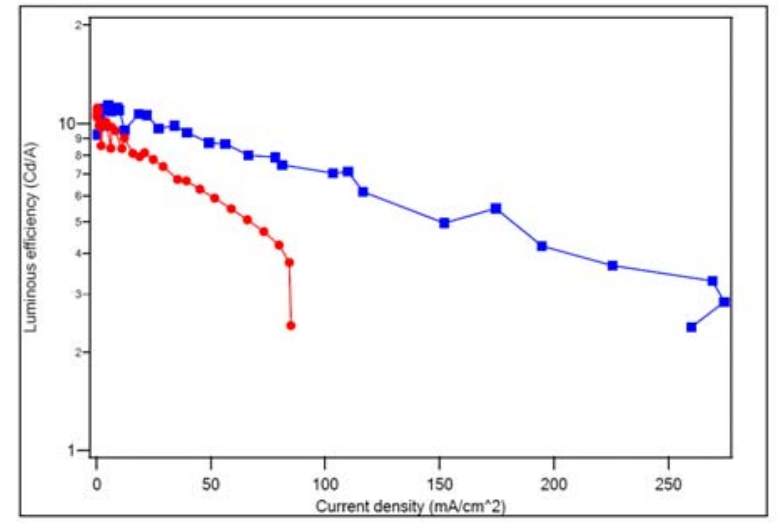

(b)

Figure 6. (a) JLV curves and (b) luminous efficiency vs current density in white SPE PhOLEDs (blue) and control devices without NPs (red).

carriers. We came to a conclusion that metal NPs have two mechanism that affect the performance of OLEDs. First, there is a variation of the emissive rates in chromophores. Second, NPs can affect charge transport properties of organic materials such as conductivity (several mechanisms are discussed below). Amazingly, both mechanisms may have a similar manifestation. For example, increased conductivity of PEDOT:PSS HTL may lead to the efficient operation of OLEDs in the high current density regime due to improved charge balance in the device. At the same time, we have acquired experimental evidence that the improvement of charge transport by metal NPs alone can not explain completely the phenomena observed and there is a significant contribution from the plasmonic effect into the operation of PhOLEDs, especially in monochromatic devices. We believe that the plasmonic enhancement of the spontaneous emission rate is the dominating phenomenon in monochromatic devices. In the alltriplet, white emitting PhOLEDs, injection of charges is the major performance limiting factor. This is probably due to the poor overlap between the plasmon band in NPs and broad-band emission of the multiple chromophores. White PhOLEDs with "good" interfaces and exhibiting high performance figures are less susceptible to the introduction of NPs. At the same time, unoptimized devices can be improved readily by enhancement of charge injection caused by addition of NPs to the device. This explains the fact that the effect of NPs addition is most prominent in batches of devices with mediocre performance. 


\section{Studies of Metal NPs Influence on the Charge Injection in PhOLEDs.}

The enhanced performance of SPE PhOLEDs can not be explained solely on the basis of the chromophore's emission rate enhancement. Addition of metal NPs to the HTL leads to a noticeable change in the device I-V curve, suggesting that metal NPs modify the charge injection and transport properties of the PhOLED. This issue was discussed during the peer-review meeting and, in order to address reviewers concerns, we have undertaken an extensive study of

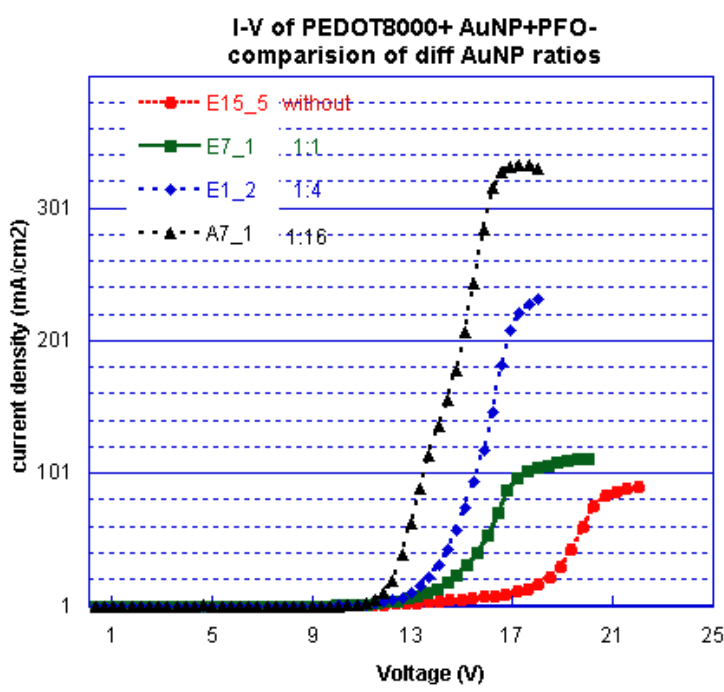

Figure 7. I-V curves in hole-only PFO devices as a function of the NPs load into HTL the metal NPs influence on the electrical characteristics of PhOLEDs. Our studies have been focused on the device architecture shown in the Figure 1. Obviously, NPs most likely affect hole injection since they are incorporated into the interface between HTL and light emitting layer. In order to avoid additional effects on the $\mathrm{I}-\mathrm{V}$ characteristics from recombination current and mutual interactions between electrons and holes, we prepared single carrier (hole only) devices by using high work function metal (gold) for the cathode deposition. High work function of gold creates a large potential barrier for the injection of electrons and the current is formed solely by holes. The complete suppression of the electron injection was confirmed by the absence of the electroluminescence even at high bias voltages. The architecture of the tested hole-only devices was ITO/PEDOT:PSS(+Au NPs)/polymer/Au. The tested polymers include PFO, PVK, and F8BT. Similar changes of the I-V characteristics have been observed in all polymer materials, that demonstrates the universal character of the phenomenon studied. To avoid additional variation of I-V curves, we maintained the constant thickness of PEDOT:PSS and polymer layers in all devices. The results of PFO-based devices testing are shown in the Figure 7. As one can see, addition of metal NPs leads to significant increase of the current density at high bias voltages when the diode is operating in the bulk charge limited regime. At the same time, the turn on voltage remains almost unchanged upon introduction of the NPs into HTL. The latter indicates that the transition from injection limited to bulk charge limited regime is not affected by the presence of NPs in the HTL. Thus, the potential barrier for the hole injection remains unchanged. The experimental data are consistent with the increase of the HTL conductivity caused by the NPs. At first glance, this is quite surprising finding, because the volume fraction of NPs in the HTL never exceeded $0.02 \%$. However, one must consider that the conductivity of gold is about $10^{7}$ times higher than that of PEDOT:PSS. If NPs form conductive bridges between ITO and organic layer, then even a small surface area occupied by NPs is sufficient for efficient current injection. Also, the phenomena observed can be explained by p-doping of PEDOT:PSS by $\mathrm{Au}$ NPs. Indeed, the workfunction of gold in the nanocrystalline form embedded into a polymer is not precisely known. Literature reports provide the value of Au workfunction in thin films in the range 5.0-5.4 eV. Thus, it is possible that Au NPs can act as shallow electron 
acceptor sites for PEDOT:PSS leading to increase of the free carriers density and, subsequently, the conductivity of HTL.

Besides being a finding of a fundamental interest, this results has a large potential for creation of highly conductive and transparent materials for the hole transport layers. Indeed, we demonstrated that a very small volume fraction of high workfunction metal NPs is sufficient to increase dramatically the injection of holes. At the same time, the low surface density of the NPs preserves the polymer layer transparency which important for the good outcoupling of the emission. Thus, by finding a proper transparent polymer material and doping it with metal NPs, one can create a charge transport material with high effective workfunction and conductivity. Additionally, the NPs can serve as a dopant free of the migration effects due to their large size on the molecular scale.

\section{Improvement of Charge Injection in Solution Processed OLEDs by Use of Ionic Species}

The simple architecture of white PhOLEDs described previously demonstrated high luminance and luminous efficiency in a simple, single emissive layer device fabricated by solution processing methods. However, for efficient illumination applications luminous and quantum efficiencies are not sufficient. Indeed, the power efficacy is the critical parameter, since it shows how efficiency the power drawn from the electrical outlet is converted into visible light. To compete with fluorecesent lighting technology, organic LEDs have to demonstrate sustained power efficacy in excess of $60 \mathrm{Lm} / \mathrm{W}$. One may notice that our best monochromatic, green PhOLEDs have the luminous efficiency close to $60 \mathrm{Cd} / \mathrm{A}$ corresponding to the internal quantum efficiency of $80 \%$. Values close to $20 \mathrm{Cd} / \mathrm{A}$ and $70 \%$, respectively, have been achieved by us in the white-emitting PhOLEDs. Thus our devices are quite efficient in terms of photons/electron. However, from analysis of L-J-V curves (where L is the luminous efficiency), one can conclude that this is the high value of the bias voltage that limits the power efficacy of the PhOLEDs. Increasing the driving voltage leads to the higher power dissipated by the device, which in its turn leads to the reduction of the power efficacy value. During the project's BP1 and BP2, we came to the conclusion that metal NPs affect the performance of white PhOLEDs primarily via improvement of the charge carriers injection and transport rather than via SP-assisted acceleration of light emission processes. Moreover, our work indicates that the problem of efficient charge injection is critical for the further development of solution processed PhOLEDs. In order to increase the efficiency of the PhOLEDs we attempted to address the problem by using charge injection/transport layers based on ionic materials (solid electrolytes).

In a simplified model, the current through the device is determined by the electronic properties of the materials, such as concentration of charge carriers, their mobility, morphology, etc., and electric field applied across the device. The most straightforward approach is then to decrease the thickness of the device in order to increase the magnitude of the applied electric field. However, this approach is not very feasible for solution-prepared devices, due to the difficulties in control over geometry of organic layers comprising the PhOLED. Interfacial phenomena play a significant role in the operation of an OLED as well. The basic models demonstrate that mismatch or unfavorable alignment of energy levels in adjacent materials create potential barriers which impede injection and transport of charge carriers. Traditionally, this problem is addressed either by using an optimal combination of materials with energy level favoring the transport of charges or by using special charge injection and charge transport layers 
which serve as a buffer smoothing the interface. Both approaches have been implemented successfully in devices fabricated by thermal deposition process which allows for a precise control over deposition of materials. The solution based methods lack such accuracy and the development of new efficient charge injection/transport materials along with optimized deposition protocols can be considered as the most straightforward solution for the reduction of the operational bias.

In our work, we decided to employ conjugated poly- and oligoelectrolytes (CPEs and COEs, respectively) for improvement of the electron injection. We have an extensive synthetic expertise and design experience of water soluble CPEs from concurrent effort by our group aimed at development of polymer-based bioassays. These materials have solubility compatible with the basic OLED architecture used in our work and can be deposited on top of organicsoluble LEL without dissolving it. Our previous work has demonstrated that CPEs can significantly improve the electron injection in MEH-PPV-based OLEDs, especially from cathodes with high value of workfunction such as Al. This provides additional benefits of increased device stability since one can use cathode metals less volatile than traditional alkali metal-based cathodes. Details of the charge injection improvement by CPEs and COEs are outside the scope of this project. We attempted to experimentally find answers for the following questions: (a) do COE and CPE provide any positive effects on the charge injection in monochromatic and white-emitting PhOLEDs; (b) what is the optimal composition of the material; (c) find the optimal deposition protocols ensuring the optimal performance of the device; (d) can CPEs and COEs be used in combination with metal NPs.

A possible mechanism of the CPEs' influence on the charge injection in organic devices can be explained by mobility of the ions embedded into CPEs. Application of the electric field to the layer of ionic species leads to the relatively slow redistribution of ionic charges which compensates the electric field inside the layer and creates large gradients of the field in the

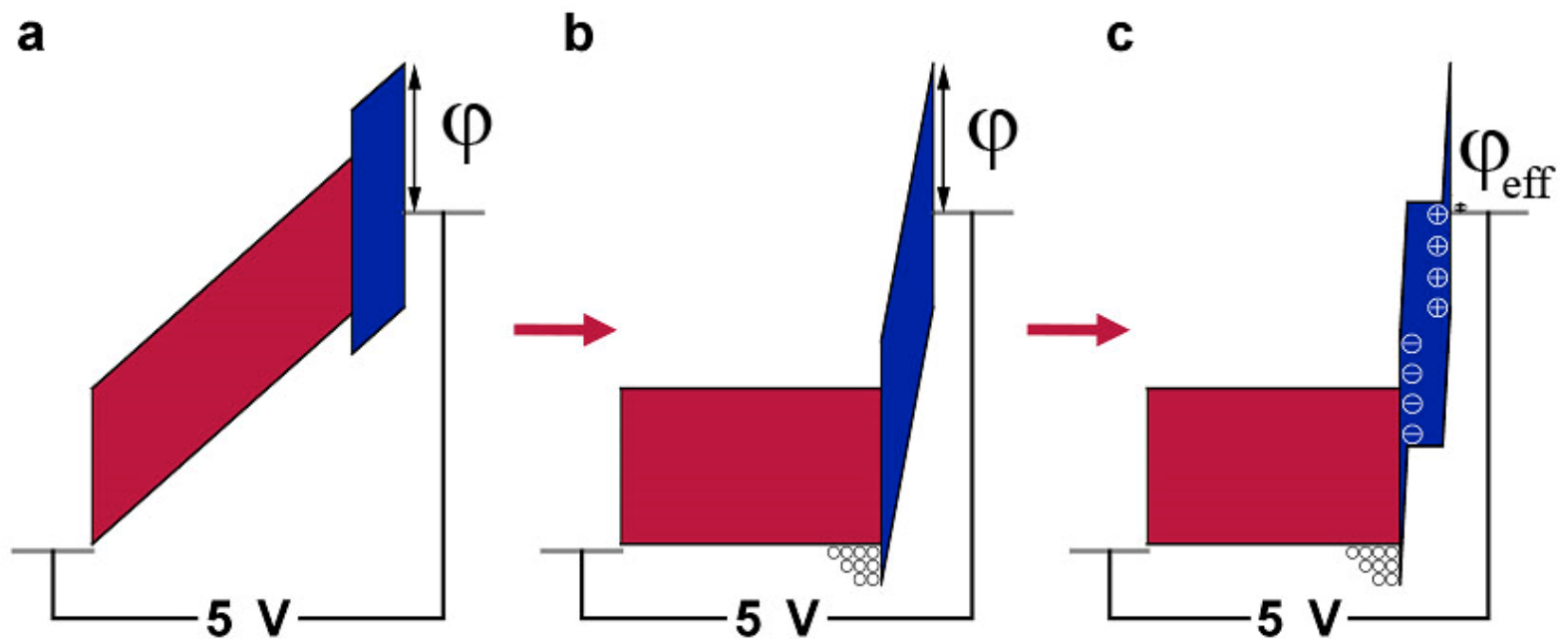

Figure 8. A possible mechanism of charge injection enhancement by CPE. (In FowlerNordheim model, $J \sim E^{2} \exp \left(-k \varphi^{3 / 2} / E\right)$, where $E$ is the electric field applied and $k$ is a constant).

interfacial regions. In the polarized state, the effective height of potential barriers is reduced thus allowing at achieve higher current densities (See Figure 8). Similar mechanism is found in the Light Emitting Cells (LECs) where redistribution of ions by the external field leads to the formation of p-i-n junctions. Since the electric field inside the ETL is close to zero, the charge 
transport is functioning in the diffusion limited regime. In such situation, the morphology and thickness of the ionic polymer layer will play a significant role. Use of highly polarizable materials allows for the use of high workfunction cathodes, such as $\mathrm{Al}$ that has been demonstrated successfully in MEH-PPV based devices.

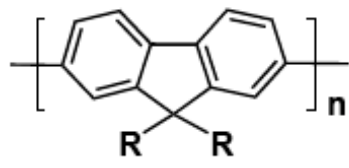

$\mathbf{R}=\left(\mathrm{CH}_{2}\right)_{6} \stackrel{\oplus}{\mathrm{N}} \mathrm{Me}_{3} \underset{\ominus}{\mathrm{X}}$<smiles>[X]C(F)(Br)Br</smiles>

(a)

(b)
Al (or Au) Cathode

Polyelectrolyte ETL

Electroluminescent polymer

ITO Glass

Figure 9. (a) Chemical structure of CPEs and (b) structure of a device with CPE ETL.

Initially, we have tested several model systems utilizing CPEs based on fluorene backbone with different pendant ionic groups. Studies were performed on DMO-PPV- and PFObased OLEDs in order to establish fabrication protocols and evaluate structure dependent properties of ETL materials. The chemical structure of the CPE is shown in Figure $9(\mathrm{a}) . \mathrm{Br}, \mathrm{CF}_{3}$, $\mathrm{SO}_{3}$, and tetrakis(imidazolyl)borate $\left(\mathrm{BIm}_{4}\right)$ anions where used as counter ions. The structure of the test devices is shown in the Figure 9(b). OLEDs with ETL utilized Al cathodes and devices with $\mathrm{Ba} / \mathrm{Al}$ cathodes where used in control groups. Without CPE ETL, devices with Al cathode did not show electroluminescence even at high bias voltage due to high potential barrier for the electron injection caused by a large difference between LUMO energy in DMO-PPV and workfunction of Al. Introduction of CPE ETL enabled electron injection form Al leading to the
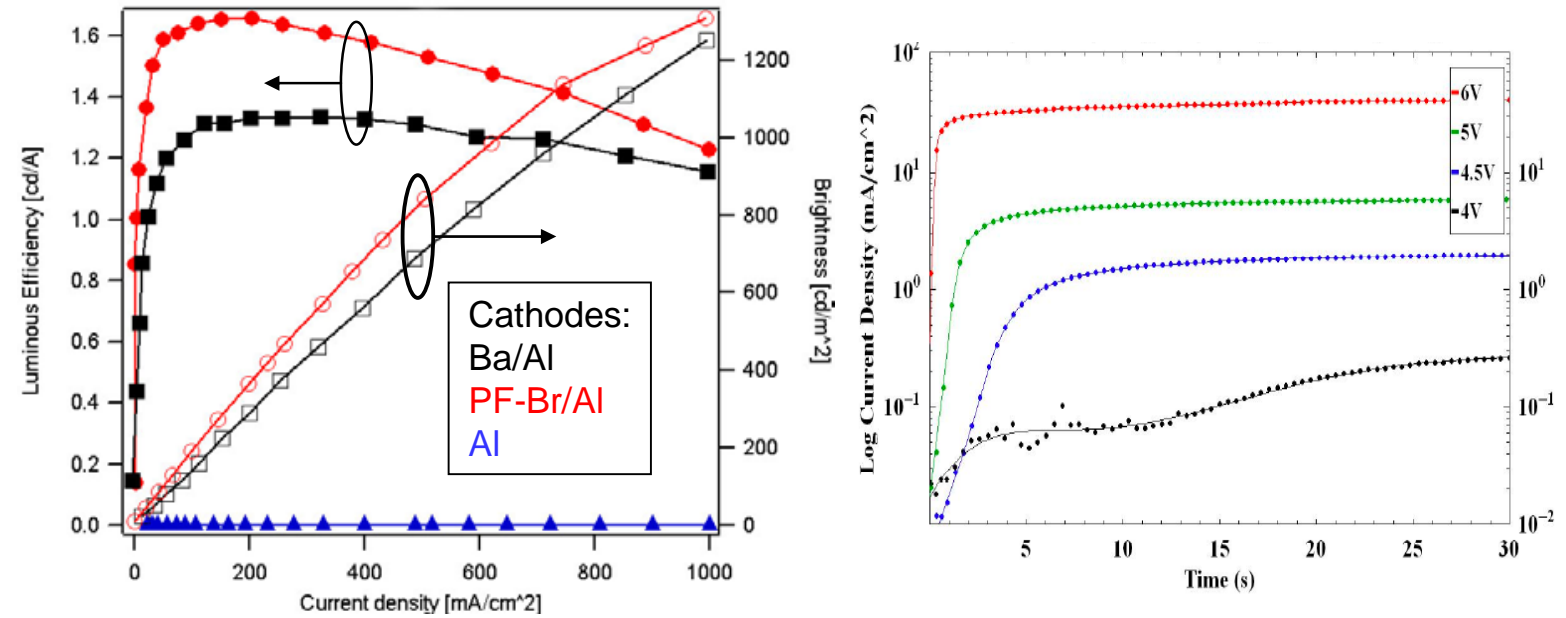

Figure 10. (a) Performance of OLEDs with ETL and different cathodes. (b) Temporal response of electroluminescence in devices with CPE ETL. 
onset of the light emission. CPE ETL provided for the higher luminous efficiency and brightness than in control devices with $\mathrm{Ba} / \mathrm{Al}$ cathode at a given bias voltage.

However, the use of CPE for improved injection of charge carriers has certain drawbacks. Devices with CPE ETL exhibit a delayed onset of the current density and electroluminescence upon application of the driving voltage (Figure 10 (b)). The delay varies depending on the composition of ETL and the voltage applied and is consistent with redistribution of ionic species within the CPE layer by the electric field. Delayed response of the OLED may be negligible in a lightning application, however free migration of charged species and their possible migration into adjacent layers may reduce the operational life-time of the device. This issue has been addressed by use of COEs which is discussed later on in this report.

After proof of principle experiments with DMO-PPV-based devices, we attempted to utilize CPE ETLs in white-emitting PhOLEDs. We utilized our standard test bed architecture ITO/PEDOT/PVK;OXD-7;FIrPic;Ir(mppy) $)_{3} ; \operatorname{Ir}($ piq)/Cathode described previously. Both Ba/Al and $\mathrm{Al}$ cathodes have been tested, and ETL has been introduced between LEL and the cathode. However, addition of CPE ETL had negative effect on the luminescent properties of LEL. Regardless of composition of ETL, a significant reduction of the luminous efficiency has been observed in comparison with control devices. Optical excitation of luminescence in devices with ETL indicated that LEL emission quenched in the presence of CPE layer. We discovered that quenching effects can be minimized by using optimized deposition procedures. Initially casting solutions had been prepared in neat methyl alcohol. Addition of water to the mixture and annealing after the deposition of ETL significantly reduced the harmful effects of ETL on the LEL quantum yield of luminescence. Neat methanol dissolves the LEL and allows diffusion of CPE into it leading to the reduced emission of the light. Addition of water reduces this effect but annealing of ETL is required to remove water from the device. We did not observe any acceleration of the device degradation on the time scale of several hours in devices with ETL deposited from methanol/water solutions and heat treated after the deposition despite even trace amount water lead to efficient quenching of the phosphor's emission. Additionally, we found that CPEs exhibit quite poor surface coverage on top of the LEL. High resolution imaging with Atomic Force Microscope (AFM) have showed that in most cases only small fraction of the LEL surface is coated with CPE. We believe that this is due to hydrophobic properties of LEL preventing adhesion of CPEs solution. Postdeposition annealing and use of optimized deposition protocols improve ETL morphology but it still remains poor in the vast majority of cases.

We have studied another class of materials for improvement of the charge carriers injection in solution processed OLEDs, namely conjugated oligoelectrolites (COEs). Relative to polymer-based materials, COEs provide better-defined molecular structures, do not exhibit molecular weight distributions, and can be obtained in higher degrees of purity. Additionally, the absence of batch-to-batch variations in their structural properties should lead to more reproducible device fabrication protocols. COE used by us utilized fluorene backbone units with ionic groups similar to use in CPE work (See Figure 11). COEs tend to form mesh-like patterns on the LEL surface similar to CPEs but respond better to the heat treatment in vacuum during the deposition of the cathode. Unlike CPEs, COEs offer almost instantaneous onset of electroluminescence upon application of the bias voltage. The reason for this is not quite clear yet despite COEs should exhibit higher ionic mobility than the CPE-based OLEDs and ion redistribution-related effects should be more pronounced in COE layers. Monodisperse chemical composition of COEs allows for better control of their properties by variation of the pendant groups and counterions. The best performing COEs utilized BIm 4 anion. Similar to CPEs, we 

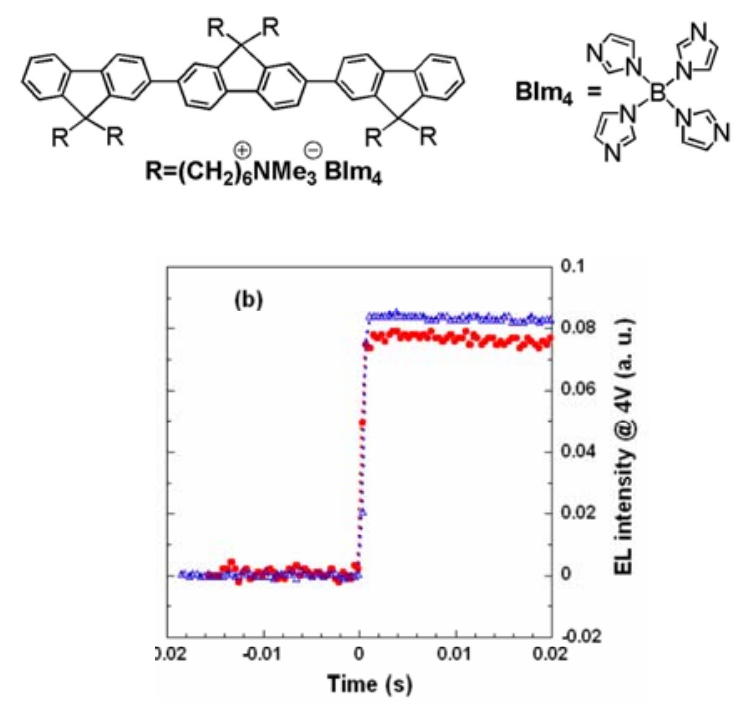

Figure 11. (a) Chemical structure of FFF$\mathrm{BIm}_{4}$. (b) Temporal response of device with $\mathrm{Ba} / \mathrm{Al}$ (blue) and $\mathrm{BIm}_{4} / \mathrm{Al}$ (red) cathode. had to optimize the composition of ETL casting solutions and use alcohol/water mixtures as a solvent. This enabled us to achieve optimal surface morphology of the ETL and minimize negative effects of the COE on emissive properties of LEL. But the major advance is related to the application of COE in whiteemitting electrophosphorescent devices. We incorporated COE ETL into white PhOLEDs described previously. In devices with ETL, Al cathodes have been used, whereas reference PhOLEDs were equipped with $\mathrm{Ba} / \mathrm{Al}$ electrode. FFF-BIm $_{4}$ COE (shown in the Figure 11) is used for the fabrication of ETL. As one can see from the Figure 12, devices without ETL and with $\mathrm{Al}$ cathode are very inefficient and exhibit high turn-on voltage if compared to control devices with Ba/Al layer. Addition of COE ETL restores the device performance to the original level or even enables one to exceed the parameter of PhOLEDs with alkali cathode. Despite that the performance increase is not large, it serves as a proof of the principle and immediately enables fabrication of solution-processed OLEDs with an environmentally stable cathode.
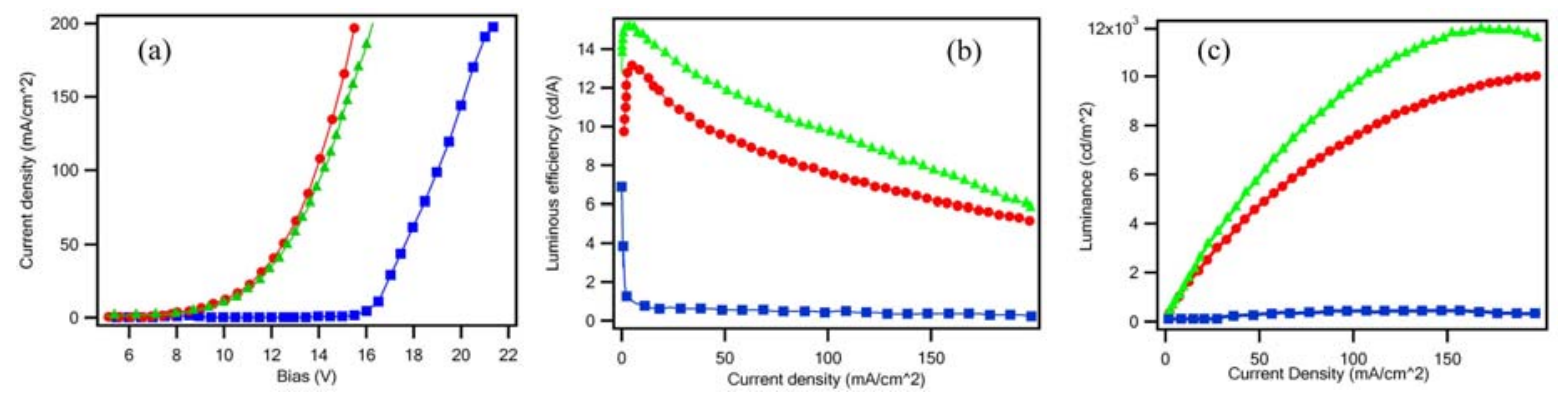

Figure 12. Performance of white-emitting, single layer PhOLEDs with COE ETL. (a) Current density - Voltage, (b) Luminous Efficiency - Current density, (c) Luminance Current Density curves in devices with $\mathrm{Al}$ (blue), $\mathrm{Ba} / \mathrm{Al}$ (red), FFF-BIm $4 / \mathrm{Al}$ (green) cathodes.

Another approach that we have explored is to use highly polarizeable small molecule materials sometimes dubbed as "zwitterions". They are expected to be less sensitive to the variation of the fabrication protocols than COEs and CPEs and can be deposited by thermal evaporation methods. The latter would enable one to extend the technology into the well developed and very large segment of small molecule OLEDs. The materials developed by us utilized the structural motif shown in the Figure 13. It consists of $\mathrm{BIm}_{4}$ core with one or several pendant groups. In our case, the pendant groups were represented by alkyl chain with variable length (1-16 carbon atoms). The number of alkyl chains and their length affect critically morphology and transport properties of ETL. The mechanism of zwitterions' influence on the charge injection is similar to that of COEs and CPEs and is attributed to the reorientation of 
molecular dipoles by applied electric field and associated increase of the local field in the vicinity of cathode/ETL and ETL/LEL interfaces.

4C16-BIm 4 and 2C16-BIm 4 zwitterions demonstrated the

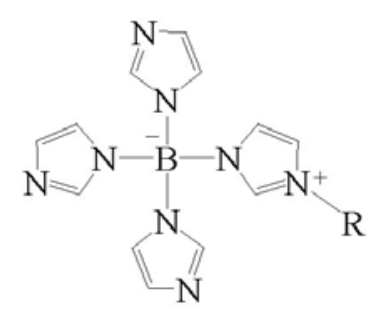

$\mathrm{R}=\mathrm{C}_{1} \mathrm{H}_{3}, \quad \mathbf{C}_{\mathbf{1}}-\mathrm{BIm}_{4}$

$\mathrm{R}=\mathrm{C}_{6} \mathrm{H}_{13}, \quad \mathbf{C}_{6}-\mathrm{BIm}_{4}$

$\mathrm{R}=\mathrm{C}_{16} \mathrm{H}_{33}, \mathrm{C}_{16}-\mathrm{BIm}_{4}$

Figure 13. Structure of a zwitterion. best performance in polyfluorene-based OLEDs. A series of devices with ETL/Al and ETL/Ba/Al cathodes has been fabricated and tested devices with ETL/Al cathodes have demonstrated performance similar to that of control OLEDs with $\mathrm{Ba} / \mathrm{Al}$ electrodes. ETL/Ba/Al devices have demonstrated a significant ( $\sim 1.7 \mathrm{~V}$ or $25 \%)$ reduction of the operational bias voltage.

A series of white emitting devices has been fabricated using PVK/PFO blend as a host material and $4 \mathrm{C} 16-\mathrm{BIm}_{4} / \mathrm{Ba} / \mathrm{Al}$ cathodes. The structure of devices was ITO/PEDOT/PVK:PFO:0.3\%Irm:0.3\%piqIr/4C16-BIm4/Ba/Al. These PhOLEDs emitted nearly perfect white-light and exhibited about $25 \%$ drop of the operational bias voltage at $1000 \mathrm{Cd} / \mathrm{m}^{2}$ as well as two-fold increase of the power efficacy (from 5 to 10 $\mathrm{Lm} / \mathrm{W}$ ) in comparison to devices without ETL. This architecture has not been fine-tuned, thus the absolute values of the power efficacy are not record high (but still quite good for an essentially single layer solution processed OLED).

\section{Task 1 Development of SP-enhanced organometallic triplet emitters.}

We decided to use only commercially available organic materials during BP2 and BP3 in order to reduce the cost of SPE PhOLEDs manufacturing and simplify their structure. Pilot white emitting devices utilized in-house synthesized phosphors, but they are currently phased out by materials available on the open market.

SPE PhOLEDs described in this report had metal NPs embedded into the HTL. In this architecture, the influence of metal NPs on the device parameters is limited by the small volume of interaction between surface plasmons and light emitters. Greater enhancement of performance is anticipated in SPE PhOLEDs with NPs incorporated directly into the light emitting layer (LEL). Some experiments with SPE PhOLEDs utilizing this architecture were performed during BP1 and demonstrated feasibility of this approach. Nevertheless, the concentration of metal NPs in the LEL in these PhOLEDs was much smaller than the optimal value and effects of NP-doping were less pronounced than in devices with NP-doped HTL. The optimal concentration of NPs in LEL can be estimated from the spatial extent of the photonic states density modification around the metal surface. From these considerations, the optimal distance between NPs centers in LEL is about 5 NP diameters. This allowed us to estimate the optimal concentration of NPs in the solutions used for the device casting as $\sim 10^{14} \mathrm{~cm}^{-1}$. Also, NPs have to have solubility compatible with the materials used in the LEL, which are usually soluble in non-polar, organic solvents. Commercially available NPs are prepared via reduction of the gold salts in aqueous medium, and are soluble only in highly polar solvents, such as water and some alcohols, and can not be admixed into solutions used for the LEL fabrication. We prepared several batches of organic soluble NPs with the surface coated with alkylamine molecules. Hydrophobic alkyl chains ensure solubility of NPs in non-polar media which can be controlled by the variation of the alkyl chain length. However, our experiments demonstrated that this approach does not allow to achieve target concentration of NPs, because at concentrations exceeding $10^{12} \mathrm{~cm}^{-1} \mathrm{NPs}$ become 
prone to the aggregation via interdigitation of alkyl chains. The aggregation is less pronounced for shorter molecules containing 6 to 8 carbon atoms, but still the maximum concentration of unaggregated NPs never exceeded $5 \times 10^{12} \mathrm{~cm}^{-1}$. We were able to achieve concentration of $10^{13}$ $\mathrm{cm}^{-1}$ in solutions of small (5 nm diameter) NPs overcoated with trioctylphosphine oxide (TOPO) but plasmonic effects in such small particles are negligible and render them ineffective in SPE PhOLED devices.

Currently, all known synthetic approaches fail to produce solutions of metal NPs in nonpolar solvents with concentration suitable for the efficient doping of the LEL. This represents a significant problem not only for SPE OLEDs development but also for the fabrication SPenhanced photovoltaic devices which require similar concentration of NPs. For this reason, we decided not to extend this work into BP3 and to concentrate our efforts on the fine tuning of the devices utilizing water soluble NPs in low concentration solutions.

NPs used in our work were acquired from commercial sources or synthesized in house if the commercial NPs did not suit our requirements. We have developed several methods of NPs synthesis and surface functionalization. The work on organic-soluble NPs led to discovery of the protocol for deposition of organic layers with improved photovoltaic performance which is currently being patented and licensed by our industrial partners.

Task 2. Theoretical modeling and analysis of experimental data on SPR-chromophore interactions.

We have conducted detailed studies of the metal NPs influence on the charge carrier injection and transport. As discussed above, introduction of the metal NPs into HTL in single carrier (hole-only) devices leads to a significant increase of the injection current density. To understand the underlying physical mechanism of the current injection enhancement, the I-V curves of the devices have been analyzed using several theoretical models, such as charge tunneling, ohmic model, and bulk charge limited injection. Results of the data analysis indicate that the former mechanisms can not be responsible for the injection current increase and the NPs do not affect the height of the potential barrier for the hole injection into LEL from HTL. This is confirmed by the constant turn-on bias voltage and similar behavior of the I-V curves at low bias voltages in devices with the different concentration of NPs. A significant increase of the current density is observed in NP-doped devices at the bias voltages well exceeding the energy of the potential barrier for the hole injection, when the device operates in the bulk charge-limited regime. The modeling indicated that the increase of the injection current is consistent with the effective increase of positive charges mobility in the hole transport layer material.

At the same time, addition of gold NPs into a water-soluble electron transport layer (ETL) located between the LEL and cathode also leads to the enhancement of the device performance in the manner consistent with the enhancement of the radiative recombination rate in the triplet emitter. However, in this case, NPs can not improve the charge injection due to the high work function of their material and can be treated as electrons traps. Thus, the enhancement of the device performance in this case can be explained purely by the plasmonic effect.

The theoretical analysis of I-V and J-L-V curves in SPE PhOLEDs led us to conclusion that their performance enhancement has dual origin. It stems both from the enhancement of the charges injection and modification of the radiative recombination rates by the metal NPs. Contributions from these two effects are superimposed constructively and can be of the same order of magnitude in devices with the NP-doped HTL. 
Also, we have studied the influence of metal NPs on emissive properties of triplet emitters such as photoluminescence quantum yield and life-time. The experiments have been conducted under photoexcitation of phosphorescence and variable amount of metal NPs has been added to the solid films containing chromophores. The data on the emission life-time and efficiency have been analyzed to prove the fundamental principles of the radiative decay technology.

\section{$\underline{\text { Status of the Project's Milestones }}$}

\section{Milestone 1.}

Demonstration of SPE PhOLED Technology

SPE PhOLEDs operation has been demonstrated during BP1.

\section{Milestone 2}

Implementation of SPE PhOLED technology in white-emitting PhOLEDs .

SPE effects have been demonstrated in white devices. However, they do not provide for a major improvement of performance. Charge injection and transport remain the limiting factors.

\section{Milestone $3^{*}$}

Development of solution-fabricated white-emitting PhOLEDs, with target luminous efficiency $20 \mathrm{Im} / \mathrm{W}$, and the color of emission on the blackbody locus.

Current maximum power efficacy $\sim 17 \mathrm{~lm} / \mathrm{W}$ in a single layer device.

Improvement of the white PhOLEDs performance by metal NP doping: 4-5 increase of the operational density, maximum luminance $80000-100000 \mathrm{Cd} / \mathrm{m}^{2}$.

Maximum luminance achieved is $\sim 50000 \mathrm{~cd} / \mathrm{m}^{2}$. Radiative life-time is not a limiting factor for the performance of white-emitting devices.

\section{Summary of Project's MajorAchievements:}

- High performance green and blue SPE PhOLEDs utilizing coinage metal NPs embedded into the charge transport layers. (Task 3)

- High performance, all-triplet, white emitting PhOLEDs fabricated via solution processing and utilizing single layer architecture. (Task 3)

- Poof-of-principle use of ionic materials (electrolytes) for improvement of charge injection in solution processed OLEDs. (Task 3)

- $25 \%$ reduction of the operational bias voltage in devices with zwitterionic electron injection layer. (Task 3)

- Understanding of multiple mechanisms of NPs influence on the device parameters (SP and charge transport related effects). (Task 2)

\footnotetext{
* Milestone 3 was adjusted after BP2 review.
} 
- $\quad$ Synthesis of organic soluble metal NPs and development of high impact technology for deposition of organic layers for PV applications. (Task 1)

\section{Acknowledgements}

The PIs are grateful to the following individuals for support and creative discussions during the project execution.

\section{UCSB:}

Prof. Alan Heeger and members of his group.

Prof. Thuc-Quyen Nguyen, Corey Hoven, Mark Dante, and other members of Nguyen group

Dupont Displays

Dr. Marc Sims

US Department of Energy

Dr. James Broderick, Dr. Ryan Egidi, Dr. Ed Petrow 


\section{Patents and publications stemming and supported by the project}

\section{Patents:}

US patent 6,999,222. Plasmon assisted enhancement of organic optoelectronic devices.

US Patent Application 11/949, Processing Additives for Improved Efficiency

\section{Journal Publications:}

"Enhancement of Phosphorescence by Surface-Plasmon Resonances in Colloidal Metal Nanoparticles: The Role of Aggregates”, Ostrowski, J.C., et. Al, Adv. Func. Mat. 2006, 16, 1221-1227.

"Efficiency enhancement in low-bandgap polymer solar cells by processing with alkane dithiols”, Peet, J. et al, Nature Materials, 2007, 6, 497-500.

"Ion Motion in Conjugated Polyelectrolyte Electron Transporting Layers", Hoven, C. et al., J. Am. Chem. Soc., 2007, 36, 10976-10977.

"Efficient Single Active Layer Electrophosphorescent White Polymer Light-Emitting Diodes”, Wu, H. B. et al, Adv. Mat., 2008, 20, 698

"Conjugated Oligoelectrolyte Electron Transport/Injection Layers for Organic Optoelectronic Devices”, Yang, R. Q. et al, J. Am. Chem. Soc., 2008, 130, 3282

"LED to LEC Transition Behavior in Polymer Light-Emitting Devices”, Shao, Y., Bazan, G. C., Heeger, A. J., Adv. Mat., 2008, 20, 1191-1193.

"Solvent Effects on the Architecture and Performance of White-Emitting Polymer LightEmitting Diodes with ConjugatedOligoelectrolyte Electron Transport Layers“, Xu, Y. et al, Adv. Mat., 2008, Accepted for publication.

“Zwitterionic self-assembled electron injection layers in Polymer Light-Emitting Diodes”, in preparation.

Conference contributions: 18 at major conferences. 


\section{Project man power matrix}

\begin{tabular}{|c|c|c|c|}
\hline Team Member & Location & Project Staffing & Project Roles \\
\hline $\begin{array}{l}\text { University } \\
\text { California, } \\
\text { Barbara }\end{array}$ & \begin{tabular}{r|l} 
of & Santa Ba \\
Santa & CA
\end{tabular} & \begin{tabular}{|l} 
Prof .Guillermo C. Bazan \\
Dr. Alexander \\
Mikhailovsky \\
Dr. Hongbin Wu \\
Dr. Omer Mermer \\
Dr. Yunhua Xu \\
Dr. Fuke Wang \\
Dr. Juhyun Park \\
Jeffrey Peet
\end{tabular} & $\begin{array}{l}\text { Principal Investigator } \\
\text { Principal Investigator } \\
\text { Postdoc (PhOLED } \\
\text { fabrication) } \\
\text { Postdoc (PhOLED } \\
\text { fabrication and testing) } \\
\text { Postdoc (PhOLED } \\
\text { fabrication and testing) } \\
\text { Synthesis of metal } \\
\text { nanoparticles } \\
\text { NEXAFS measurements } \\
\text { Graduate student (PhOLEDs, } \\
\text { synthesis, materials } \\
\text { characterization) }\end{array}$ \\
\hline
\end{tabular}

